\title{
How COVID-19 affects peripartum women's mental health
}

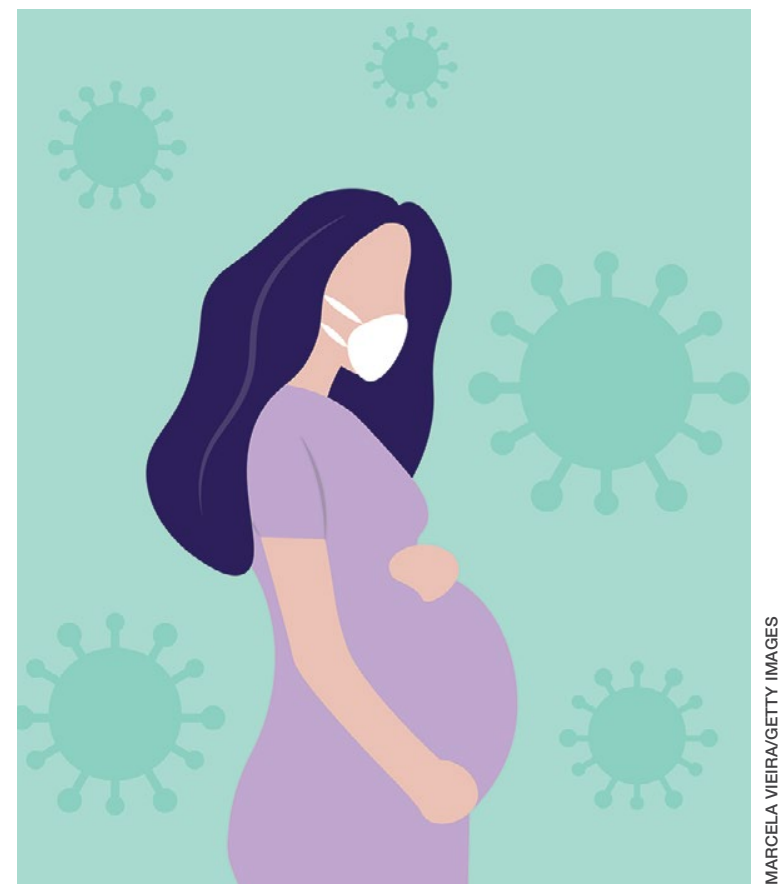

\section{Briana Tillman, DO, IBCLC}

PGY-2 Psychiatry Resident

Department of Psychiatry

The Medical Center of Aurora

Aurora, Colorado

\section{Nicholas Sloan, DO}

PGY-1 Psychiatry Resident

Department of Psychiatry

The Medical Center of Aurora

Aurora, Colorado

\section{Patricia Westmoreland, MD}

Medical Director, The Women's Unit

Psychiatry Residency Program Director

The Medical Center of Aurora

Aurora, Colorado

\section{Addressing the factors that increase risk can help reduce anxiety, depression, and stress}

$\mathrm{T}$ he COVID-19 pandemic has had a negative impact on the mental health of people worldwide, and a disproportionate effect on peripartum women. In this article, we discuss the reasons for this disparity, review the limited literature on this topic, and suggest strategies to safeguard the mental health of peripartum women during the COVID-19 pandemic.

\section{Catastrophic events and women's mental health}

During the peripartum period, women have increased psychosocial and physical health needs. ${ }^{1}$ In addition, women are disproportionately affected by natural disasters and catastrophic events, ${ }^{2}$ which are predictors of psychiatric symptoms during the peripartum period. ${ }^{3}$ Mass tragedies previously associated with maternal stress include wildfires, hurricanes, migrations, earthquakes, and tsunamis. ${ }^{4,5}$ For example, pregnant women who survived severe exposure during Hurricane Katrina (ie, feeling that one's life was in danger, experiencing illness or injury to self or a family member, walking through floodwaters) in 2005 had a significantly increased risk of developing posttraumatic stress disorder (PTSD) and depression compared with pregnant women who did not have such exposure. ${ }^{6}$ After the 2011 Tōhoku earthquake and tsunami in Japan, the prevalence of

\section{Disclosures}

The authors report no financial relationships with any companies whose products are mentioned in this article, or with manufacturers of competing products. This research was supported (in whole or in part) by HCA Healthcare and/or an HCA Healthcare-affiliated entity. The views expressed in this article represent those of the authors and do not necessarily represent the official views of HCA Healthcare or any of its affiliated entities. 
psychological distress in pregnant women increased, especially among those living in the area directly affected by the tsunami. ${ }^{5}$

Epidemics and pandemics also can adversely affect peripartum women's mental health. Studies conducted before the COVID-19 pandemic found that previous infectious disease outbreaks such as severe acute respiratory syndrome (SARS), the 2009 influenza A (H1N1) pandemic, and Zika had negative emotional impacts on pregnant women. ${ }^{7}$ Our review of the limited literature published to date suggests that COVID-19 is having similar adverse effects.

\section{COVID-19 poses both medical and psychiatric threats}

COVID-19 infection is a physical threat to pregnant women who are already vulnerable due to the hormonal and immunological changes inherent to pregnancy. A metaanalysis of 39 studies with a total of 1,316 pregnant women indicated that the most frequently reported symptoms of COVID-19 infection were cough, fever, and myalgias. ${ }^{8}$ However, COVID-19 infection during pregnancy is also associated with an increase in pregnancy complications and adverse birth outcomes. ${ }^{9}$ According to the CDC, compared with their nonpregnant counterparts, pregnant women are at greater risk for severe COVID-19 infection and adverse birth outcomes such as preterm birth. ${ }^{10}$ Pregnant women who are infected with severe acute respiratory syndrome coronavirus 2 (SARSCoV-2; the virus responsible for COVID-19) risk ICU admission, caesarean section, and perinatal death. ${ }^{8}$ A Swedish study of 2,682 pregnant women found an increase in preeclampsia among women who tested positive for SARS-CoV-2, a finding attributed to COVID-19's pattern of systemic effects. ${ }^{11}$ Vertical transmission of the novel coronavirus from mother to fetus appears to be rare but possible. ${ }^{12}$

In addition to the physical dangers of becoming infected with COVID-19, the perceived threat of infection is an added source of anxiety for some peripartum women. In addition to the concerns involved in any pregnancy, COVID-19-related sources of distress for pregnant women include worrying about harm to the fetus during pregnancy, the possibility of vertical transmission, and exposures during antenatal appointments, during employment, or from a partner. ${ }^{8,13}$

The death toll from factors associated with COVID-19 adds to the mental health burden. For every person who dies of COVID-19, an estimated 9 others may develop prolonged grief or PTSD due to the loss of someone they loved. ${ }^{14,15}$ A systematic review found that PTSD in the perinatal period is associated with negative birth and child outcomes, including low birth weight and decreased rates of breastfeeding. ${ }^{16}$ The COVID-19 pandemic has disrupted human interactions, from social distancing rules and lockdowns of businesses and social activities to panic buying of grocery staples and increased economic insecurity. ${ }^{1}$ These changes have been accompanied by a rise in mental health challenges. For example, according to an August 2020 CDC survey, $40.9 \%$ of US adults reported at least 1 adverse mental or behavioral health condition, including symptoms of anxiety or depression (30.9\%), symptoms of a trauma- and stressor-related disorder related to the pandemic (26.3\%), and having started or increased substance use to cope with stress or emotions related to COVID-19 $(13.3 \%) .{ }^{17}$

COVID-19-related traumas and stressors appear to affect women more than men. A study from China found that compared with men, women had significantly higher levels of self-reported pandemic-related anxiety, depression, and posttraumatic stress symptoms (PTSS). ${ }^{18}$ This trend has been observed in other parts of the world. A study conducted by the UK Office of National Statistics reported anxiety levels were $24 \%$ higher in women vs men as reflected by scores on a self-rated anxiety scale. ${ }^{19}$

Many factors influence the disproportionate impact of COVID-19 on women in general, and peripartum women in particular (Box,,$^{20-26}$ page 20).

\section{Psychiatric diagnoses in peripartum women}

Multiple studies and meta-analyses have begun to assess the impact of the COVID-19

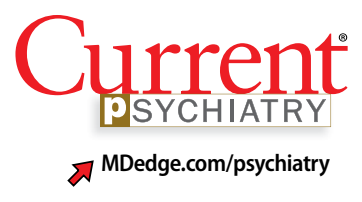

\section{Clinical Point \\ COVID-19 infection during pregnancy is associated with increased pregnancy complications and adverse birth outcomes}

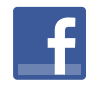

Discuss this article at www.facebook.com/ MDedgePsychiatry 


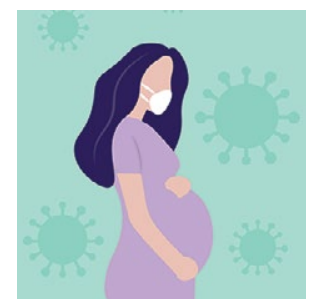

COVID-19 and peripartum women

\section{Clinical Point}

COVID-19
significantly
increases the risk
of anxiety and
depression in
women during the
peripartum period

\section{How COVID-19 disproportionally affects women}

Eactors that predispose women to increased

stress during COVID-19 include an increase in child care burdens brought about by school closures and subsequent virtual schooling. ${ }^{20}$ Intimate partner violence has spiked globally during COVID-19 restrictions. ${ }^{24}$ Women also represent the majority of the health care workforce (76\%) and often take on informal caregiving roles; both of these roles have seen increased burdens during the pandemic. ${ }^{25}$ Already encumbered by prepandemic gender pay inequalities, women are filing unemployment claims at a significantly increased rate compared to men. ${ }^{26}$

For women of childbearing age, the disruption of routine clinical care during
COVID-19 has decreased access to reproductive health care, resulting in increases in unintended pregnancies, unsafe abortions, and deaths. ${ }^{20}$ Another source of stress for pregnant women during COVID-19 is feeling unprepared for birth because of the pandemic, a phenomenon described as "preparedness stress." 21 Visitor restriction policies and quarantines have also caused women in labor to experience birth without their support partners, which is associated with increased posttraumatic stress symptoms. ${ }^{22}$ These restrictions also may be associated with an increase in women choosing out-of-hospital births despite the increased risk of adverse outcomes. ${ }^{23}$ pandemic on maternal mental health. One meta-analysis of 8 studies conducted in 5 countries determined that COVID-19 significantly increases the risk of anxiety in women during the peripartum period. ${ }^{27}$ Results of another meta-analysis of 23 studies with $>24,000$ participants indicated that the prevalence of anxiety, depression, and insomnia in peripartum women was significantly higher during the pandemic than in pre-pandemic times. ${ }^{28}$

In an online survey of 4,451 pregnant women in the United States, nearly onethird of respondents reported elevated levels of pandemic-related stress as measured by the newly-developed PandemicRelated Pregnancy Stress Scale. ${ }^{3}$ The rates were even higher among women who were already at risk for elevated stress levels, such as those who had survived abuse, those giving birth for the first time, or those experiencing high-risk pregnancies. ${ }^{3}$ Living in a pandemic "hot spot" also appeared to impact peripartum stress levels.

COVID-19 has adverse effects on women's mental health specifically during the postpartum period. One study from a center in Italy found a high prevalence of depressive symptoms and PTSS in the postpartum period, with COVID-19-related factors playing an "indirect role" compared with prenatal experiences and other individual factors. ${ }^{2}$ A British study of mothers of infants age $\leq 12$ months found that traveling for work, the impact of lockdown on food affordability, and having an income of less than $£ 30,000$ per year (approximately $\$ 41,000)$ predicted poorer mental health during the pandemic. ${ }^{29}$ Results of a study from China indicated that more than one-quarter of pregnant and postpartum women experienced depression during the pandemic, and women who worried about infection risk or missing pediatric visits were at increased risk. ${ }^{30}$

\section{How to mitigate these risks}

The increase in pandemic-related mental health concerns in the general population and specifically in peripartum women is a global health care challenge. Investing in mitigation strategies is necessary not only to address the current pandemic, but also to help prepare for the possibility of future traumatic events, such as another global pandemic.

For pregnant women, ensuring access to outdoor space, increasing participation in healthy activities, and minimizing disruptions to prenatal care can protect against pandemic-related stress. ${ }^{3}$ Physical activity is an effective treatment for mild to moderate depressive symptoms. Because of the significant decrease in exercise among pregnant women during the pandemic, encouraging safe forms of physical activity such as online fitness classes could improve mental health outcomes for these patients. ${ }^{27}$ When counseling peripartum 
women, psychiatrists need to be creative in recommending fitness interventions to target mood symptoms, such as by suggesting virtual or at-home programs.

In an online survey, 118 obstetricians called for increased mental health resources for peripartum women, such as easier access to a helpline, educational videos, and mental health professionals. ${ }^{13}$ Increased screening for psychiatric disorders throughout the peripartum period can help identify women at greater risk, and advancements in telepsychiatry could help meet the increased need for psychiatric care during COVID-19. Psychiatrists and other mental health clinicians should consider reaching out to their colleagues who specialize in women's health to establish new partnerships and create teams of multidisciplinary professionals.

Similarly, psychiatrists should familiarize themselves with telehealth services available to peripartum patients who could benefit from such services. Telehealth options can increase women's access to peripartum care for both medical and psychiatric illnesses. Online options such as women's support groups, parenting classes, and labor coaching seminars also represent valuable virtual tools to strengthen women's social supports.

Women who need inpatient treatment for severe peripartum depression or anxiety might be particularly reluctant to receive this care during COVID-19 due to fears of becoming infected and of being separated from their infant and family while hospitalized. Clinicians should remain vigilant in screening peripartum women for mood disorders that might represent a danger to mothers and infants, and not allow concerns about COVID-19 to interfere with recommendations for psychiatric hospitalizations,

\section{Related Resources}

- Hu YJ, Wake M, Saffery R. Clarifying the sweeping consequences of COVID-19 in pregnant women, newborns, and children with existing cohorts. JAMA Pediatr. 2021; 75(2):117-118. doi: 10.1001/jamapediatrics.2020.2395

- Tomfohr-Madsen LM, Racine N, Giesbrecht GF, et al. Depression and anxiety in pregnancy during COVID-19: a rapid review and meta-analysis. Psychiatry Res. 2021; 300:113912. doi: 10.1016/j.psychres.2021.113912

when necessary. The creation of small, women-only inpatient behavioral units can help address this situation, especially given the possibility of frequent visits with infants and other peripartum support. Investment into such units is critical for supporting peripartum mental health, even in nonpandemic times.

What about vaccination? As of mid-May 2021, no large clinical trials of any COVID19 vaccine that included pregnant women had been completed. However, 2 small preliminary studies suggested that the mRNA vaccines are safe and effective during pregnancy. ${ }^{31,32}$ When counseling peripartum patients on the risks and benefits, clinicians need to rely on this evidence, animal trials, and limited data from inadvertent exposures during pregnancy. While every woman will weigh the risks and benefits for her own circumstances, the CDC, the American College of Obstetricians and Gynecologists, and the Society for MaternalFetal Medicine have all stated that the mRNA vaccines should be offered to pregnant and breastfeeding individuals who are eligible for vaccination. ${ }^{33}$ Rasmussen et $\mathrm{al}^{33}$ have published a useful resource for clinicians regarding COVID-19 vaccination and pregnant women.

continued

\section{Bottom Line}

During the COVID-19 pandemic, peripartum women have experienced increased rates of anxiety, depression, and stress. Psychiatric clinicians can help these patients by remaining vigilant in screening for psychiatric disorders, encouraging them to engage in activities to mitigate COVID-19's adverse psychological effects, and referring them to care via telehealth and other resources as appropriate.

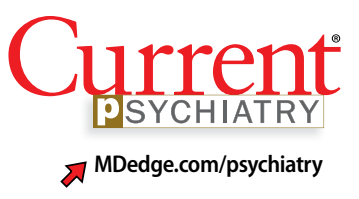

Clinical Point

Telehealth options can increase women's access to peripartum care for both medical and psychiatric illnesses 


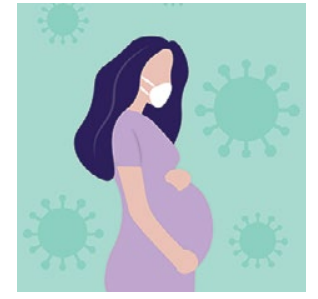

COVID-19 and peripartum women

\section{Clinical Point}

\section{The CDC and}

several professional

organizations state

that $\mathrm{mRNA}$ vaccines

should be offered to

eligible pregnant or

breastfeeding women

\section{References}

1. Chivers BR, Garad RM, Boyle JA, et al. Perinatal distress during COVID-19: thematic analysis of an online parenting forum. J Med Internet Res. 2020;22(9):e22002. doi: 10.2196/ 22002

2. Ostacoli L, Cosma S, Bevilacqua F, et al. Psychosocial factors associated with postpartum psychological distress during the Covid-19 pandemic: a cross-sectional study. BMC Pregnancy Childbirth. 2020;20(1):703. doi: 10.1186/s12884020-03399-5

3. Preis H, Mahaffey B, Heiselman C, etal. Vulnerability and resilience to pandemic-related stress among U.S. women pregnant at the start of the COVID-19 pandemic. Soc Sci Med. 2020;266:113348. doi: 10.1016/j.socscimed. 2020.113348

4. Olson DM, Brémault-Phillips S, King S, et al. Recent Canadian efforts to develop population-level pregnancy intervention studies to mitigate effects of natural disasters and other tragedies. J Dev Orig Health Dis. 2019;10(1):108114. doi: $10.1017 /$ S2040174418001113

5. Watanabe Z, Iwama N, Nishigori H, et al. Japan Environment \& Children's Study Group. Psychological distress during pregnancy in Miyagi after the Great East Japan Earthquake: the Japan Environment and Children's Study. J Affect Disord. 2016;190:341-348. doi: 10.1016/j.jad.2015.10.024

6. Xiong X, Harville EW, Mattison DR, et al. Hurricane Katrina experience and the risk of post-traumatic stress disorder and depression among pregnant women. Am J Disaster Med. 2010;5(3):181-187. doi: 10.5055/ajdm.2010.0020

7. Brooks SK, Weston D, Greenberg N. Psychological impact of infectious disease outbreaks on pregnant women: rapid evidence review. Public Health. 2020;189:26-36. doi: 10.1016/j.puhe.2020.09.006

8. Diriba K, Awulachew E, Getu E. The effect of coronavirus infection (SARS-CoV-2, MERS-CoV, and SARS-CoV) during pregnancy and the possibility of vertical maternal-fetal transmission: a systematic review and meta-analysis. Eur J Med Res. 2020;25(1):39. doi: 10.1186/s40001-020-00439-w

9. Qi M, Li X, Liu S, et al. Impact of the COVID-19 epidemic on patterns of pregnant women's perception of threat and its relationship to mental state: a latent class analysis. PLoS One. 2020;15(10):e0239697. doi: 10.1371/journal.pone.0239697

10. Centers for Disease Control and Prevention. Investigating the impact of COVID-19 during pregnancy. Updated February 4, 2021. Accessed April 29, 2021. https://www. cdc.gov/coronavirus/2019-ncov/cases-updates/specialpopulations/pregnancy-data-on-covid-19/what-cdc-isdoing.html

11. Ahlberg M, Neovius M, Saltvedt S, et al. Association of SARS-CoV-2 test status and pregnancy outcomes. JAMA. 2020;324(17):1782-1785. doi: 10.1001/jama.2020.19124

12. Ashraf MA, Keshavarz P, Hosseinpour P, et al. Coronavirus disease 2019 (COVID-19): a systematic review of pregnancy and the possibility of vertical transmission. J Reprod Infertil. 2020;21(3):157-168

13. Nanjundaswamy MH, Shiva L, Desai G, et al. COVID-19related anxiety and concerns expressed by pregnant and postpartum women-a survey among obstetricians. Arch Womens Ment Health. 2020; 23(6):787-790. doi: 10.1007/ s00737-020-01060-w

14. Verdery AM, Smith-Greenaway E, Margolis R, et al. Tracking the reach of COVID-19 kin loss with a bereavement multiplier applied to the United States. Proc Natl Acad Sci U SA. 2020;117(30):17695-17701. doi: 10.1073/pnas.2007476117

15. Simon NM, Saxe GN, Marmar CR. Mental health disorders related to COVID-19-related deaths. JAMA. 2020;324(15):1493-1494. doi: 10.1001/jama.2020.19632

16. Cook N, Ayers S, Horsch A. Maternal posttraumatic stress disorder during the perinatal period and child outcomes: a systematic review. J Affect Disord. 2018;225:18-31. doi: 10.1016/j.jad.2017.07.045
17. Czeisler MÉ, Lane RI, Petrosky E, et al. Mental health, substance use, and suicidal ideation during the COVID-19 pandemic - United States, June 24-30, 2020. MMWR Morb Mortal Wkly Rep. 2020;69(32):1049-1057. doi:10.15585/ mmwr.mm6932a1

18. Almeida M, Shrestha AD, Stojanac D, et al. The impact of the COVID-19 pandemic on women's mental health. Arch Womens Ment Health. 2020;23(6):741-748. doi:10.1007/ s00737-020-01092-2

19. Office for National Statistics. Personal and economic well-being in Great Britain: May 2020. Published May 4 2020. Accessed April 23, 2021. https://www.ons.gov.uk/ peoplepopulationandcommunity/wellbeing/bulletins/ personalandeconomicwellbeingintheuk/may2020

20. Kuehn BM. COVID-19 halts reproductive care for millions of women. JAMA. 2020;324(15):1489. doi: 10.1001/ jama.2020.19025

21. Preis H, Mahaffey B, Lobel M. Psychometric properties of the Pandemic-Related Pregnancy Stress Scale (PREPS). J Psychosom Obstet Gynaecol. 2020;41(3):191-197. doi: 10.1080/0167482X.2020.1801625

22. Hermann A, Fitelson EM, Bergink V. Meeting maternal mental health needs during the COVID-19 pandemic JAMA Psychiatry. 2020;78(2):123-124. doi: 10.1001/ jamapsychiatry.2020.1947

23. Arora KS, Mauch JT, Gibson KS. Labor and delivery visitor policies during the COVID-19 pandemic: balancing risks and benefits. JAMA. 2020;323(24):2468-2469. doi: 10.1001/ jama.2020.7563

24. Bradbury-Jones C, Isham L. The pandemic paradox: the consequences of COVID-19 on domestic violence. J Clin Nurs. 2020;29(13-14):2047-2049. doi: 10.1111/jocn.15296

25. Connor J, Madhavan S, Mokashi M, et al. Health risks and outcomes that disproportionately affect women during the Covid-19 pandemic: a review. Soc Sci Med. 2020;266:113364 doi: 10.1016/j.socscimed.2020.113364

26. Scharff X, Ryley S. Breaking: some states show alarming spike in women's share of unemployment claims. The Fuller Project. Accessed April 23, 2021. https:/ / fullerproject. org/story/some-states-shows-alarming-spike-in-womensshare-of-unemployment-claims/

27. Hessami K, Romanelli C, Chiurazzi M, et al. COVID-19 pandemic and maternal mental health: a systematic review and meta-analysis. J Matern Fetal Neonatal Med. 2020;1-8. doi: 10.1080/14767058.2020.1843155

28. Yan H, Ding Y, Guo W. Mental health of pregnan and postpartum women during the coronavirus disease 2019 pandemic: a systematic review and meta-analysis. Front Psychol. 2020;11:617001. doi: 10.3389/fpsyg.2020.617001

29. Dib S, Rougeaux E, Vázquez-Vázquez A, et al. Maternal mental health and coping during the COVID-19 lockdown in the UK: data from the COVID-19 New Mum Study. Int J Gynaecol Obstet. 2020;151(3):407-414. doi: 10.1002/ ijgo.13397

30. Bo HX, Yang Y, Chen J, et al. Prevalence of depressive symptoms among Chinese pregnant and postpartum women during the COVID-19 pandemic. Psychosom Med. 2020. doi: 10.1097/PSY.0000000000000904

31. Collier AY, McMahan K, Yu J, et al. Immunogenicity of COVID-19 mRNA vaccines in pregnant and lactating women. JAMA. 2021. doi:10.1001/jama.2021.7563

32. Shanes ED, Otero S, Mithal LB, et al. Severe acute respiratory syndrome coronavirus 2 (SARS-CoV-2) vaccination in pregnancy: measures of immunity and placental histopathology. Obstet Gynecol. 2021. doi: 10.1097/ AOG.0000000000004457

33. Rasmussen SA, Kelley CF, Horton JP, et al. Coronavirus disease 2019 (COVID-19) vaccines and pregnancy: what obstetricians need to know. Obstet Gynecol. 2021;137(3):408414. doi: 10.1097/AOG.0000000000004290 